\title{
Basic Sets of Special Monogenic Polynomials in Fréchet Modules
}

\author{
Gamal Farghaly Hassan, ${ }^{1,2}$ Lassaad Aloui, ${ }^{3}$ and Allal Bakali ${ }^{1}$ \\ ${ }^{1}$ Department of Mathematics, Faculty of Sciences, Northern Border University, P.O. Box 1321, Arar, Saudi Arabia \\ ${ }^{2}$ Faculty of Science, University of Assiut, Assiut 71516, Egypt \\ ${ }^{3}$ Department of Mathematics, Faculty of Sciences of Tunis, University of Tunis-El Manar, Tunis, Tunisia
}

Correspondence should be addressed to Gamal Farghaly Hassan; gamal6new@yahoo.com

Received 20 June 2016; Revised 31 October 2016; Accepted 18 December 2016; Published 14 February 2017

Academic Editor: Konstantin M. Dyakonov

Copyright (c) 2017 Gamal Farghaly Hassan et al. This is an open access article distributed under the Creative Commons Attribution License, which permits unrestricted use, distribution, and reproduction in any medium, provided the original work is properly cited.

\begin{abstract}
This article is concerned with the study of the theory of basic sets in Fréchet modules in Clifford analysis. The main aim of this account, which is based on functional analysis consideration, is to formulate criteria of general type for the effectiveness (convergence properties) of basic sets either in the space itself or in a subspace of finer topology. By attributing particular forms for the Fréchet module of different classes of functions, conditions are derived from the general criteria for the convergence properties in open and closed balls. Our results improve and generalize some known results in complex and Clifford setting concerning the effectiveness of basic sets.
\end{abstract}

\section{Introduction}

The theory of bases in function spaces plays an important role in mathematics and its applications, for example, in approximation theory, partial differential equations, geometry, and mathematical physics.

The subject of basic sets of polynomials in one complex variable, in its classical form, was introduced by Whittaker $[1,2]$ who laid down the definition of basic sets, basic series, and effectiveness of basic sets. Many well-known polynomials such as Laguerre, Legendre, Hermite, Bernoulli, Euler, and Bessel polynomials form simple bases of polynomials (see [3-7]). A significant advance was contributed to the subject by Cannon $[8,9]$ who obtained necessary and sufficient conditions for the effectiveness of basic sets for classes of functions with finite radius of regularity and entire functions.

The theory of basic sets of polynomials can be generalized to higher dimensions in several different ways, for instance, to several complex variables or to hypercomplex analysis.

The theory of basic sets of polynomials in several complex variables was developed at the end of the 1950s by Mursi and Maker [10] and later by Nassif [11] and was studied in more detail afterwards by others (c.f [12-15]). Also, the representation of matrix functions by bases of polynomials has been studied by Makar and Fawzy [16]. For more information about the study of basic sets of polynomials in complex analysis, we refer to [17-20].

In theory of basic sets of polynomials in hypercomplex analysis, Abul-Ez and Constales gave in $[21,22]$ the extension of the theory of bases of polynomials in one complex variable to the setting of Clifford analysis. This is the natural generalization of complex analysis to Euclidean space of dimension larger than two, where the holomorphic functions have values in Clifford algebra and are null solutions of a linear differential operator. An important subclass of the Clifford holomorphic functions called special monogenic functions is considered, for which a Cannon theorem on the effectiveness in closed and open ball $[21,23]$ was established. Many authors studied the basic sets of polynomials in Clifford analysis [2430].

In [31], Adepoju laid down a treatment of the subject of basic sets of polynomials of a single complex variable in Banach space which is based on functional analysis considerations. Also, the authors in $[12,32]$ studied the basic sets of polynomials of several complex variables in Banach space.

We shall lay down in this paper a treatment of the subject of basic sets based primarily on functional analysis and Clifford analysis. The aim of this treatment is to construct a criterion, of general type, for effectiveness of basic sets in Fréchet modules. By attributing particular forms to these Fréchet 
modules, we derive, in the remaining articles of the present paper, from the general criterion of effectiveness already obtained, particular conditions for effectiveness in the different forms of the regions which are relevant to our subsequent work. Thus, effectiveness in open and closed balls is studied. In addition, we give some applications of the effectiveness of basic sets of polynomials in approximation theory concerned with

(1) the expansion of Clifford valued functions in closed and open ball by infinite series in a given sequence of basic sets,

(2) the expansion of Clifford valued functions in closed and open ball by infinite series in a given sequence of Cannon sets of special monogenic polynomials.

These new results extend and generalize the known results in complex and Clifford setting given in [12, 21, 23, 31, 32].

\section{Notation and Preliminaries}

In order to introduce our results, we give several notations and assumptions.

Let us denote by $\left\{e_{1}, \ldots, e_{m}\right\}$ the canonical basis of the Euclidean vector space $\mathbb{R}^{m}$ and by $\mathscr{A}_{m}$ the associated real Clifford algebra in which one has the multiplication rules $e_{i} e_{j}+e_{j} e_{i}=-2 \delta_{i j}, i, j=1, \ldots, m$, where $\delta_{i j}$ denotes the Kronecker symbol.

A vector space basis for the Clifford algebra $\mathscr{A}_{m}$ is given by the set $\left\{e_{A}: A \subseteq\{1, \ldots, m\}\right\}$ with $e_{A}=e_{\alpha_{1}, \ldots, \alpha_{h}}=$ $e_{\alpha_{1}} \cdots e_{\alpha_{h}}, 1 \leq \alpha_{1}<\alpha_{2}<\cdots<\alpha_{h} \leq m$, and $e_{\phi}=e_{0}=1$. Every $a \in \mathscr{A}_{m}$ can be written in the form $a=\sum_{A} a_{A} e_{A}$ with $a_{A} \in \mathbb{R}$. The conjugate element of $a \in \mathscr{A}_{m}$ is defined by $\bar{a}=\sum_{A} a_{A} \bar{e}_{A}$, where $\bar{e}_{A}=\bar{e}_{\alpha_{h}} \cdots \bar{e}_{\alpha_{1}}, \bar{e}_{j}=-e_{j}(j=1, \ldots, m)$, and $\bar{e}_{0}=e_{0}=1$.

We denote also by $\mathbb{R}^{m+1}=\operatorname{span}_{\mathbb{R}}\left\{e_{0}, e_{1}, \ldots, e_{m}\right\}=\mathbb{R} \oplus$ $\mathbb{R}^{m} \subset \mathscr{A}_{m}$ the space of paravectors $x=x_{0} e_{0}+\sum_{j=1}^{m} x_{j} e_{j}$. In this notation, the paravector $x$ will be represented in the form $x=x_{0}+\underline{x}$ with $\mathrm{Sc}(x)=x_{0}$ being the scalar part and $\operatorname{Vec}(x)=\underline{x}$ being the vector part of $x$. The induced Clifford norm of arbitrary $a \in \mathscr{A}_{m}$ is given by $|a|=\left(\sum_{A}\left|a_{A}\right|^{2}\right)^{1 / 2}$.

Some care must be taken when using this norm to estimate product. We will always use the formula $|a b| \leq$ $2^{m / 2}|a||b|$.

One useful approach to generalize complex analysis to higher dimensional spaces is the Cauchy-Riemann approach which is based on the consideration of functions that are in the kernel of the generalized Cauchy-Riemann operator $D=\sum_{i=0}^{m} e_{i}\left(\partial / \partial x_{i}\right)$ in $\mathbb{R}^{m+1}$ (for more details, see $[33,34]$ ).

Definition 1 (unitary right $\mathscr{A}_{m}$-module). A unitary right $\mathscr{A}_{m^{-}}$ module $X$ is an abelian group $(X,+)$ with a mapping $X \times$ $\mathscr{A}_{m} \rightarrow X ;(f, \lambda) \rightarrow f \lambda$ such that for all $\lambda, \mu \in \mathscr{A}_{m}$ and $f, g \in X:$
(i) $f(\lambda+\mu)=f \lambda+f \mu$.
(ii) $f(\lambda \mu)=(f \lambda) \mu$.
(iii) $(f+g) \lambda=f \lambda+g \lambda$.
(iv) $f e_{0}=f$.

Remark 2. Notice that $X$ becomes a real vector space if $\mathbb{R}$ is identified with $\mathbb{R} e_{0}=\mathscr{A}_{0} \subset \mathscr{A}_{m}$.

In the following, all $\mathscr{A}_{m}$-modules will be right $\mathscr{A}_{m}$ modules.

Definition 3 ( $\mathscr{A}_{m}$-linear operator). Let $X$ and $Y$ be two unitary $\mathscr{A}_{m}$-modules. Then a function $T: X \rightarrow Y$ is said to be an $\mathscr{A}_{m}$-linear operator if, for all $f, g \in X$ and $\lambda, \mu \in \mathscr{A}_{m}$,

$$
T(f \lambda+g \mu)=T(f) \lambda+T(g) \mu .
$$

The set of all $\mathscr{A}_{m}$-linear operators from $X$ into $Y$ is denoted by $L(X, Y)$.

Definition 4 (proper system of seminorms). Let $X$ be a unitary $\mathscr{A}_{m}$-module. Then a family $\mathscr{P}$ of functions $p: X \rightarrow$ $[0, \infty)$ is said to be a proper system of seminorms on $X$ if the following conditions are fulfilled:

$\left[P_{1}\right]$ There exists a constant $C_{0} \geq 1$ such that, for all $p \in \mathscr{P}$, $\lambda \in \mathscr{A}_{m}$ and $f, g \in X:$

(i) $p(f+g) \leq p(f)+p(g)$

(ii) $p(f \lambda) \leq C_{0}|\lambda| p(f)$, and $p(f \lambda)=|\lambda| p(f)$ if $\lambda \epsilon$ $\mathbb{R}$.

$\left[P_{2}\right]$ For any finite number $p_{1}, p_{2}, \ldots, p_{k} \in \mathscr{P}$, there exist $p \in \mathscr{P}$ and $C>0$ such that, for all $f \in X$,

$$
\sup _{j=1,2, \ldots, k} p_{j}(f) \leq C p(f) .
$$

$\left[P_{3}\right]$ If $p(f)=0$ for all $p \in \mathscr{P}$ then $f=0$.

Definition 5 (Fréchet module). A Fréchet module $E$ over $\mathscr{A}_{m}$ is a Hausdorff space with a countable proper system of seminorms $\mathscr{P}=\left(p_{i}\right)_{i \geq 0}$ satisfying

(i) $i<j \Rightarrow p_{i}(f) \leq p_{j}(f) ;(f \in E)$,

(ii) a subset $U$ of $E$ is open if, $\forall f \in U$, there exist $\epsilon>0$ and $N \geq 0$ such that

$$
\left\{g \in E: p_{i}(f-g) \leq \epsilon\right\} \subset U, \quad \forall i \leq N
$$

(iii) $E$ is complete with respect to this topology.

We denote by $\mathscr{T}_{E}$ the topology defined by the family $\mathscr{P}$ of seminorms on $E$.

Definition 6 (convergent sequences in the topology $\mathscr{T}_{E}$ ). The sequence $\left(f_{k}\right)_{k \geq 0}$ of elements of $E$ converges in the topology $\mathscr{T}_{E}$ to the element $g$ of $E$, if and only if, for all $p_{i} \in \mathscr{P}$, we have

$$
\lim _{k \rightarrow \infty} p_{i}\left(f_{k}-g\right)=0
$$

We may also equivalently say that the sequence $\left(f_{k}\right)_{k \geq 0}$ converges in $E$ to $g$ with respect to $\mathscr{T}_{E}$. 
It is a familiar property for the Fréchet module $E$ that a seminorm $\sigma$ on $E$ is $\mathscr{T}_{E}$-continuous, if and only if there is a seminorm $p_{i} \in \mathscr{P}$ and a positive finite constant $C_{i}$ such that

$$
\sigma(f) \leq C_{i} p_{i}(f) ; \quad(\forall f \in E) .
$$

It is also known that a linear operator $L$ on $E$ is continuous if and only if there is a seminorm $p_{i} \in \mathscr{P}$ and a constant $K_{i}$ such that

$$
|L(f)| \leq K_{i} p_{i}(f) ; \quad(\forall f \in E) .
$$

\section{Basis and Absolute Basis}

Definition 7 (basis for Fréchet module $E$ ). Let $E$ be a Fréchet module over $\mathscr{A}_{m}$. A sequence $\left(z_{n}\right)_{n \geq 0}$ of nonzero elements of $E$ is called a basis for $E$ if, for each element $f \in E$, there is one and only one sequence $\left(Z_{n}(f)\right)_{n \geq 0}$ of the Clifford algebra $\mathscr{A}_{m}$, such that

$$
f=\sum_{n=0}^{\infty} z_{n} Z_{n}(f) .
$$

Definition 8 (Cauchy's inequality). We shall assume that Cauchy's inequality holds for the basis $\left(z_{n}\right)_{n \geq 0}$ in the form that for each $p_{i} \in \mathscr{P}$ there is a positive finite constant $M_{i}$ such that

$$
\left|Z_{n}(f)\right| p_{i}\left(z_{n}\right) \leq M_{i} p_{i}(f)
$$

for all integers $n$ and for all $f \in E$.

Also, the nature of the problems considered here necessitates that whenever $i<j$, there is a finite positive constant $M_{i, j}$, such that

$$
\sum_{n=0}^{\infty} \frac{p_{i}\left(z_{n}\right)}{p_{j}\left(z_{n}\right)}=M_{i, j}
$$

Definition 9 (absolute basis for Fréchet module $E$ ). The basis $\left(z_{n}\right)_{n \geq 0}$ is called an absolute basis for $E$ if the series

$$
\sum_{n=0}^{\infty}\left|Z_{n}(f)\right| p_{i}\left(z_{n}\right)
$$

is convergent in $\mathbb{R}$ for all integers $i$ and for all $f \in E$. Thus, in this case, we can write

$$
\sum_{n=0}^{\infty}\left|Z_{n}(f)\right| p_{i}\left(z_{n}\right)=Q_{i}(f)<\infty ; \quad(i \geq 0, f \in E) .
$$

We start with the following introductory theorems.

Theorem 10. If $\left(z_{k}\right)_{k \geq 0}$ is a basis for $E$ and if Cauchy's inequality (8) is satisfied, then $Z_{n}$ is a continuous linear operator on $E$, orthonormal to $\left(z_{k}\right)_{k \geq 0}$.

Proof. It easily follows from the uniqueness of representation (7) that if $f, g \in E$ and $\lambda, \mu \in \mathscr{A}_{m}$, then

$$
Z_{n}(f \lambda+g \mu)=Z_{n}(f) \lambda+Z_{n}(g) \mu,
$$

so that $Z_{n}$ is a linear operator on $E$. Also, putting $f=z_{k}$ in (7), it can be verified that

$$
Z_{n}\left(z_{k}\right)=\delta_{n}^{k}
$$

and $Z_{n}$ is orthonormal to $\left(z_{k}\right)_{k \geq 0}$.

We deduce the continuity of $Z_{n}$ from (6) and (8).

Theorem 11. Let $\left(z_{n}\right)_{n \geq 0}$ be an absolute basis for $E$ and let $Q_{i}$ be given by (11). Then the family $\left(Q_{i}\right)_{i \geq 0}$ forms a proper system of continuous seminorms. Moreover, for $i<j$, there exists a constant $N_{i, j}$ such that

$$
Q_{i}(f) \leq N_{i, j} p_{j}(f)
$$

for all $f \in E$.

Proof. Firstly, we prove that the family $\left(Q_{i}\right)_{i \geq 0}$ is a proper system of seminorms as follows.

$\left[P_{1}\right]$ We observe, from the linearity of $Z_{n}$ and properties (i) and (ii) of seminorms, that

$$
\begin{aligned}
Q_{i}(f+g) & =\sum_{n=0}^{\infty}\left|Z_{n}(f+g)\right| p_{i}\left(z_{n}\right) \\
& =\sum_{n=0}^{\infty}\left|Z_{n}(f)+Z_{n}(g)\right| p_{i}\left(z_{n}\right) \\
& \leq \sum_{n=0}^{\infty}\left\{\left|Z_{n}(f)\right|+\left|Z_{n}(g)\right|\right\} p_{i}\left(z_{n}\right) \\
& =Q_{i}(f)+Q_{i}(g), \\
Q_{i}(f \lambda) & \leq C_{0}|\lambda| Q_{i}(f), \quad C_{0} \geq 1,
\end{aligned}
$$

whenever $f, g \in E$ and $\lambda \in \mathscr{A}_{m}$.

$\left[P_{2}\right]$ Let $Q_{1}, Q_{2}, \ldots, Q_{k}$ be defined by (11). Since $\mathscr{P}$ is a proper system of seminorms, then there exist $p \in \mathscr{P}$ and $C>$ 0 such that, for all $f \in X$,

$$
\begin{aligned}
\sup _{1 \leq j \leq k} Q_{j}(f) & \leq \sum_{n=0}^{\infty}\left|Z_{n}(f)\right| \sup _{1 \leq j \leq k} p_{j}\left(z_{n}\right) \\
& \leq C \sum_{n=0}^{\infty}\left|Z_{n}(f)\right| p\left(z_{n}\right)=C Q(f) .
\end{aligned}
$$

$\left[P_{3}\right]$ Suppose that $Q_{i}(f)=0 \forall i$; then $\left|Z_{n}(f)\right| p_{i}\left(z_{n}\right)=$ $0 \forall i, n$. Since $\mathscr{P}$ is a proper system of seminorms and $\left(z_{k}\right)_{k \geq 0}$ is a basis, for each $n \in \mathbb{N}$ there exists $j$ such that $p_{j}\left(z_{n}\right) \neq 0$. So $Z_{n}(f)=0$ for all $n \in \mathbb{N}$ and hence (7) implies that $f=0$.

Finally, when $i<j$, inequality (14) can be obtained from (8), (9), and (11) as follows:

$$
\begin{aligned}
Q_{i}(f) & =\sum_{n=0}^{\infty}\left|Z_{n}(f)\right| p_{i}\left(z_{n}\right) \\
& =\sum_{n=0}^{\infty}\left|Z_{n}(f)\right| p_{j}\left(z_{n}\right) \frac{p_{i}\left(z_{n}\right)}{p_{j}\left(z_{n}\right)} \leq M_{j} M_{i, j} p_{j}(f) \\
& :=N_{i, j} p_{j}(f) .
\end{aligned}
$$


It follows from condition (5) that the seminorm $Q_{i}$ is continuous on $E$. Therefore the family $\left(Q_{i}\right)_{i \geq 0}$ forms a proper system of continuous seminorms, as required.

\section{Basic Sets}

In this section, we lay down the definition of basic sets, basic coefficients, and basic series and show (in Theorem 13) that when the basic series converges, it will converge to the element with which it is associated.

Let $\left(P_{k}\right)_{k \geq 0}$ be a sequence of nonzero elements of $E$, and suppose that $\left(\pi_{k}\left(z_{n}\right)\right)_{n, k \geq 0}$ is a matrix of coefficients in the Clifford algebra $\mathscr{A}_{m}$ such that, for each $n \geq 0$, we have the unique representation

$$
z_{n}=\sum_{k=0}^{\infty} P_{k} \pi_{k}\left(z_{n}\right) .
$$

In this case, we shall call the sequence $\left(P_{k}\right)_{k \geq 0}$ a basic set on E.

Let $f$ be any element of $E$ and substitute (18) in (7) to obtain the formal series

$$
f \sim \sum_{k=0}^{\infty} P_{k} \Pi_{k}(f)
$$

where

$$
\Pi_{k}(f)=\sum_{n=0}^{\infty} \pi_{k}\left(z_{n}\right) Z_{n}(f) .
$$

When series (20) converges in $\mathscr{A}_{m}, \Pi_{k}(f)$ exists and is called the $k$ th basic coefficient of $f$ relative to the set $\left(P_{k}\right)_{k \geq 0}$. When the basic coefficient $\Pi_{k}(f)$ exists for all $k$, series (19) is called the basic series associated with $f$.

The following theorem is concerned with the basic coefficients $\left(\Pi_{k}(f)\right)_{k \geq 0}$.

Theorem 12. If $\Pi_{k}(f)$ is defined for all $f$ in $E$, the map $\Pi_{k}$ : $E \rightarrow \mathscr{A}_{m}$ is a continuous linear operator on $E$.

Proof. Let

$$
T_{N}(f)=\sum_{n=0}^{N} \pi_{k}\left(z_{n}\right) Z_{n}(f) .
$$

It is clear that $T_{N}$ is a continuous linear operator on $E$ as a finite sum of continuous linear functional $Z_{n}$.

Now, if $\Pi_{k}(f)$ is defined for all $f$ in $E$, the sequence $\left(T_{N}(f)\right)_{N \geq 0}$ converges pointwise to $\Pi_{k}(f)$ in $E$. Therefore, by the Banach-Steinhaus theorem for Fréchet space [35], we deduce that $\Pi_{k}$ is equally a continuous linear operator on $E$, and the theorem is established.

We now write

$$
B_{n}(f)=\sum_{k=0}^{n} P_{k} \Pi_{k}(f)
$$

for the $n$th partial sum of basic series (19). The following theorem establishes the required conformity of the limit of $B_{n}(f)$ with the space $E$.
Theorem 13. If, for every $f \in E, B_{n}(f)$ is defined for all $n$ and if the sequence $\left(B_{n}(f)\right)_{n \geq 0}$ converges in $E$ to some limit $B(f)$, then $B(f)=f$ for all elements $f \in E$.

Proof. We prove that $B$ is a continuous linear operator on $E$ as a limit of finite sum of continuous linear operators as in Theorem 12 .

Now, it can be proven, from (13) and (20), that

$$
\Pi_{k}\left(z_{n}\right)=\pi_{k}\left(z_{n}\right) ; \quad(n, k \geq 0) .
$$

Hence, (18) and (22) together yield

$$
B\left(z_{n}\right)=\sum_{k=o}^{\infty} P_{k} \pi_{k}\left(z_{n}\right)=z_{n} ; \quad(n \geq 0) .
$$

Let $f$ be any element of $E$ and write

$$
g_{k}=\sum_{j=o}^{k} z_{j} Z_{j}(f)
$$

Then, in view of (24) and (25), we have

$$
B\left(g_{k}\right)=g_{k}
$$

and, by continuity of $B$, we deduce that $B(f)=f$ and Theorem 13 is therefore established.

\section{Effectiveness of Basic Sets}

We have seen that when $\left(B_{n}(f)\right)_{n \geq 0}$ converges for each $f$ of $E, B_{n}(f)$ converges to $f$. This means that basic series (19) associated with the element $f$ converges to $f$ itself for all $f \in E$. In this case, we say that the set $\left(P_{k}\right)_{k \geq 0}$ is effective for $E$. To find a necessary and sufficient condition for the effectiveness of a basic set $\left(P_{k}\right)_{k \geq 0}$ for the space $E$, we consider, for each seminorm $p_{i} \in \mathscr{P}$, the mapping $q_{i}: E \rightarrow \mathbb{R}$ defined by

$$
q_{i}(f)=\sup _{\mu, \nu} p_{i}\left\{\sum_{k=\mu}^{v} P_{k} \Pi_{k}(f)\right\}, \quad f \in E .
$$

Suppose that $q_{i}(f)$ is finite for all $f \in E$. We first show that $q_{i}$ is a seminorm on $E$.

Let $f, g \in E$ and $\lambda \in \mathscr{A}_{m}$; it follows from (27) and the linearity of $\Pi_{k}$ that

$$
\begin{gathered}
q_{i}(f+g) \leq q_{i}(f)+q_{i}(g), \\
q_{i}(f \lambda) \leq C_{0}|\lambda| q_{i}(f) .
\end{gathered}
$$

The first result concerning the effectiveness of basic set $\left(P_{k}\right)_{k \geq 0}$ is the following theorem.

Theorem 14. For $\left(P_{k}\right)_{k \geq 0}$ to be effective for $E$, it is necessary and sufficient that, for each $p_{i} \in \mathscr{P}$, the seminorm $q_{i}$ exist and be continuous. 
Proof.

Necessity. When the basic set $\left(P_{k}\right)_{k \geq 0}$ is effective for $E$, basic series (19) associated with each element $f \in E$ converges to $f$, and it follows that $B_{n}$ is a continuous linear operator on $E$.

Therefore, if we write

$$
C_{\nu, \mu}(f)=B_{\nu}(f)-B_{\mu-1}(f) ; \quad(\nu \geq \mu>0),
$$

then from (27) we shall have

$$
q_{i}(f)=\sup _{\mu, \nu} p_{i}\left\{C_{\nu, \mu}(f)\right\} .
$$

If $\mu \rightarrow \infty$, then $C_{v, \mu}(f) \rightarrow 0$. Then for every $\epsilon>0$ there exists a natural number $m$, such that if $\mu \geq m$, then $p_{i}\left(C_{\nu, \mu}(f)\right)<\epsilon$. Hence, we have

$$
q_{i}(f) \leq \sup \left\{\sup _{\mu \leq m, v \geq \mu} p_{i}\left\{C_{\nu, \mu}(f)\right\}, \epsilon\right\} .
$$

If $\nu \rightarrow \infty$ with fixed $\mu$, then $C_{v, \mu}(f) \rightarrow f-B_{\mu-1}(f)$. Hence, for some $\epsilon>0$, there is $N$ such that, for all $\nu>N$ and $\mu \leq m$,

$$
\begin{gathered}
p_{i}\left\{C_{\nu, \mu}(f)-\left(f-B_{\mu-1}(f)\right)\right\}<\epsilon, \\
q_{i}(f) \leq \sup \left\{\sup _{\nu \leq N, \mu \leq m} p_{i}\left\{C_{\nu, \mu}(f)\right\},\right. \\
\left.\sup _{\mu \leq m} p_{i}\left\{f-B_{\mu-1}(f)\right\}+\epsilon, \epsilon\right\} .
\end{gathered}
$$

This shows that the seminorm $q_{i}$ exists.

Now, to prove the continuity of $q_{i}$, let $\left(f_{n}\right)_{n \geq 0}$ be a sequence in $E$ which converges to an element $f \in E$. By this hypothesis, if $\epsilon>0$, there exists a natural number $M$ such that if $n>M$, then $p_{i}\left(f_{n}-f\right)<\epsilon$.

Hence, if $K(\epsilon)=\sup \{M, N, m\}$, it follows that if $n \geq K(\epsilon)$, then

$$
\begin{aligned}
& q_{i}\left(f_{n}-f\right) \leq \sup \left\{\sup _{\nu \leq N, \mu \leq m} p_{i}\left\{C_{\nu, \mu}\left(f_{n}-f\right)\right\},\right. \\
& \left.\quad p_{i}\left\{\left(f_{n}-f\right)-B_{\mu-1}\left(f_{n}-f\right)\right\}+\epsilon, \epsilon\right\} \\
& \leq \sup \{\epsilon, 3 \epsilon, \epsilon\}=3 \epsilon .
\end{aligned}
$$

Thus $\lim _{n \rightarrow \infty} q_{i}\left(f_{n}\right)=q_{i}(f)$ and $q_{i}$ is a continuous seminorm on $E$.

Sufficiency. We observe from (22) and (27) that

$$
p_{i}\left\{B_{n}(f)\right\} \leq q_{i}(f) ; \quad(n \geq 0) .
$$

Since $q_{i}$ is continuous on $E$, we deduce that the sequence $\left(B_{n}\right)_{n \geq 0}$ is an equicontinuous sequence on $E$.

Now define the subspace $Y$ of $E$ by

$$
\begin{aligned}
Y:= & \left\{g \in Y, \text { the sequence }\left(B_{n}(g)\right)_{n \geq 0}\right. \\
& \text { is a Cauchy sequence }\} .
\end{aligned}
$$

It follows from the equicontinuity of $\left(B_{n}\right)_{n \geq 0}$ that the set $Y$ is closed. Hence, the set $Y \subset E$ is everywhere dense and is closed, so that $Y=\bar{Y}=E$. Therefore $\left(B_{n}(f)\right)_{n \geq 0}$ is a Cauchy sequence on $E$ and since $E$ is complete, the sequence $\left(B_{n}(f)\right)_{n \geq 0}$ converges for all $f \in E$ and hence it converges to $f$ in $E$. Thus, the set $\left(P_{k}\right)_{k \geq 0}$ is effective for $E$ and Theorem 14 is established.

Theorem 15. Suppose that $\left(z_{n}\right)_{n \geq 0}$ is an absolute basis for E. Then the basic set $\left(P_{k}\right)_{k \geq 0}$ will be effective for $E$ if and only if, for any continuous seminorm $p_{i} \in \mathscr{P}$, there is a continuous seminorm $p_{j} \in \mathscr{P}$ and a positive finite number $K_{i, j}$ such that

$$
q_{i}\left(z_{n}\right) \leq K_{i, j} p_{j}\left(z_{n}\right) ; \quad \forall n \in \mathbb{N} .
$$

Proof.

Necessity. If the basic set $\left(P_{k}\right)_{k \geq 0}$ is effective for $E$, then, by Theorem 14, the application $q_{i}$ is a continuous seminorm on $E$. Hence, by (5), there is a seminorm $p_{j} \in \mathscr{P}$ and a positive number $K_{i, j}$ such that

$$
q_{i}(f) \leq K_{i, j} p_{j}(f) ; \quad \forall f \in E .
$$

Putting $f=z_{n}, n \geq 0$, we obtain (36).

Sufficiency. Multiplying the basic coefficient $\Pi_{k}(f)$ of (20) by $P_{k}$ and using (11), (27), and (36), we obtain

$$
\begin{aligned}
& p_{i}\left\{\sum_{k=\mu}^{v} P_{k} \Pi_{k}(f)\right\} \\
& \leq C_{0} \sum_{n=0}^{\infty} p_{i}\left\{\sum_{k=\mu}^{v} P_{k} \Pi_{k}\left(z_{n}\right)\right\}\left|Z_{n}(f)\right| \\
& \leq C_{0} \sum_{n=0}^{\infty} q_{i}\left(z_{n}\right)\left|Z_{n}(f)\right| \leq C_{0} K_{i j} \sum_{n=0}^{\infty} p_{j}\left(z_{n}\right)\left|Z_{n}(f)\right| \\
& \quad=C_{0} K_{i j} Q_{j}(f),
\end{aligned}
$$

where $Q_{j}(f)$ is defined by (11). According to inequality (14), there is a seminorm $p_{l} \in \mathscr{P}$ and a positive number $M_{j l}$ such that

$$
\begin{aligned}
q_{i}(f) & =\sup _{\mu, \nu} p_{i}\left\{\sum_{k=\mu}^{v} P_{k} \Pi_{k}(f)\right\} \leq C_{0} K_{i j} Q_{j}(f) \\
& \leq C_{0} K_{i j} M_{j l} p_{l}(f), \quad \forall f \in E .
\end{aligned}
$$

It follows from condition (5) that $q_{i}$ is continuous on $E$. Then, by Theorem 14, we deduce that the set $\left(P_{k}\right)_{k \geq 0}$ is effective for $E$ and the proof of Theorem 15 is therefore terminated.

\section{Alternative Treatment of the Problem}

In this treatment, we consider the Fréchet module $E$ as a subspace of a Banach module $F$ with a continuous norm $\sigma$ such that

$$
\sigma(f) \leq p_{i}(f), \quad \forall i \in \mathbb{N}, \forall f \in E
$$


where $\mathscr{P}=\left(p_{i}\right)_{i \geq 0}$ is the family of seminorms defined, as before, in the space $E$. Thus, the topology induced in $E$ by the topology $\mathscr{T}_{F}$ of $F$ determined by the norm $\sigma$ is coarser than the topology $\mathscr{T}_{E}$ defined on $E$ by the family $\mathscr{P}$ of seminorms.

Let $\left(z_{k}\right)_{k \geq 0}$ be a basis for $E$ and let $\left(P_{k}\right)_{k \geq 0}$ be a sequence of nonzero elements of $F$. We suppose that $\left(\pi_{k}\left(z_{n}\right)\right)_{n, k \geq 0}$ is a matrix of $\mathscr{A}_{m}$ such that, for each $n \geq 0$, we have the unique representation

$$
z_{n}=\sum_{k=0}^{\infty} P_{k} \pi_{k}\left(z_{n}\right)
$$

and the convergence is in $F$. In this case, we call the sequence $\left(P_{k}\right)_{k \geq 0}$ a basic set on $F$. Let $f$ be an element of $E$ and substitute (41) in (7) to obtain the formal series

$$
f \sim \sum_{k=0}^{\infty} P_{k} \Pi_{k}(f),
$$

where

$$
\Pi_{k}(f)=\sum_{n=0}^{\infty} \pi_{k}\left(z_{n}\right) Z_{n}(f) .
$$

When series (43) converges in $\mathscr{A}_{m}$, we call $\Pi_{k}(f)$ the basic coefficient of $f$, and when $\Pi_{k}(f)$ exists for each $k$, series (42) is called the basic series of $f$. Recall that the partial sum $B_{n}$ : $E \rightarrow F$ is defined (see (22)) by

$$
B_{n}(f)=\sum_{k=0}^{n} P_{k} \Pi_{k}(f) .
$$

Theorem 12 remains unchanged, while the alternative form of Theorem 13 is the following.

Theorem 16. If, for every $f \in E, B_{n}(f)$ is defined for all $n$ and if the sequence $\left(B_{n}(f)\right)_{n \geq 0}$ converges in $F$ to some element $B(f)$, then $B(f)=f$ for all elements $f \in E$.

Proof. We prove, as before, that, for each integer $n, B_{n}$ is a linear operator from $E$ to $F$. We show now that $B_{n}$ is continuous. In fact, if $\left(f_{m}\right)_{m \geq 0}$ is a sequence of elements of $E$ converging to an element $g$ of $E$, it can be deduced from (40) and (44) and Theorem 12 that

$$
\lim _{m \rightarrow \infty} \sigma\left\{B_{n}\left(f_{m}\right)-B_{n}(g)\right\}=0 .
$$

Hence $B_{n}\left(f_{m}\right)$ converges to $B_{n}(g)$ in $F$ and hence $B_{n}: E \rightarrow F$ is a continuous linear operator. Proceeding exactly as in the proof of Theorem 13, we can deduce that $B: E \rightarrow F$ is a continuous linear operator.

Now, set $g_{k}=\sum_{j=o}^{k} z_{j} Z_{j}(f)$. It is clear that $g_{k} \rightarrow f$ in $E$ and $B\left(g_{k}\right)=g_{k}$; hence $B(f)=f$, as required.

We see that when $\left(B_{n}(f)\right)_{n \geq 0}$ converges in $F$, for every element $f \in E, B_{n}(f)$ converges in $F$ to $f$. This means that basic series (42) associated with the element $f$ converges in $F$ to the element $f$, for all $f \in E$. In this case, we say that the basic set $\left(P_{k}\right)_{k \geq 0}$ is effective for $E$ in $F$.
The necessary and sufficient condition for effectiveness of $\left(P_{k}\right)_{k \geq 0}$ for $E$ in $F$ is obtained through the expression

$$
q^{\sigma}(f)=\sup _{\mu, \nu} \sigma\left\{\sum_{k=\mu}^{\nu} P_{k} \Pi_{k}(f)\right\} ; f \in E .
$$

It can be proven in exactly the same way as before that $q^{\sigma}$ is a seminorm on $E$. The revised version of Theorem 14 is as follows.

Theorem 17. For the basic set $\left(P_{k}\right)_{k \geq 0}$ to be effective for $E$ in $F$, it is necessary and sufficient that $q^{\sigma}$ exist and be continuous on E.

Proof.

Necessity. Since $B_{n}$ is continuous linear operator from $E$ to $F$, we apply the same method as in the proof of Theorem 14.

Sufficiency. We see here, from (22) and (46), that

$$
\sigma\left(B_{n}(f)\right) \leq q^{\sigma}(f) ; \quad \forall f \in E .
$$

Since $q^{\sigma}$ is continuous on $E$, then the sequence $\left(B_{n}\right)_{n \geq 0}$ will be equicontinuous from $E$ to $F$. The proof is then completed in exactly the same way as in the proof of Theorem 14 .

Now, if $\left(z_{n}\right)_{n \geq 0}$ is an absolute basis for $E$, the effectiveness of the set $\left(P_{k}\right)_{k \geq 0}$ for $E$ in $F$ will be estimated through the expression $q^{\sigma}\left(z_{n}\right)$ as it is seen from the following Theorem which is the alternative form of Theorem 15 .

Theorem 18. Suppose that $\left(z_{n}\right)_{n \geq 0}$ is an absolute basis for $E$. Then the basic set $\left(P_{k}\right)_{k>0}$ will be effective for $E$ in $F$ if and only if there is a seminorm $p_{i} \in \mathscr{P}$ and a constant $K_{i}$ such that

$$
q^{\sigma}\left(z_{n}\right) \leq K_{i} p_{i}\left(z_{n}\right) ; \quad \forall n \geq 0 .
$$

Proof.

Necessity. If $\left(P_{k}\right)_{k \geq 0}$ is effective for $E$ in $F$, then, by Theorem 17, $q^{\sigma}$ is a continuous norm on $E$. Hence, by (5), there is a seminorm $p_{i} \in \mathscr{P}$ and a constant $K_{i}$ such that

$$
q^{\sigma}(f) \leq K_{i} p_{i}(f) ; \quad \forall f \in E .
$$

Putting $f=z_{n}$, we obtain (48).

Sufficiency. As in the proof of Theorem 15, we deduce from (11), (43), (46), and (48) that

$$
\begin{aligned}
& \sigma\left\{\sum_{k=\mu}^{v} P_{k} \Pi_{k}(f)\right\} \\
& \leq C_{0} \sum_{n=0}^{\infty} \sigma\left\{\sum_{k=\mu}^{v} P_{k} \Pi_{k}\left(z_{n}\right)\right\}\left|Z_{n}(f)\right| \\
& \leq C_{0} \sum_{n=0}^{\infty} q^{\sigma}\left(z_{n}\right)\left|Z_{n}(f)\right| \leq C_{0} K_{i} \sum_{n=0}^{\infty} p_{i}\left(z_{n}\right)\left|Z_{n}(f)\right| \\
& =C_{0} K_{i} Q_{i}(f) .
\end{aligned}
$$


Applying inequality (14), we obtain

$$
q^{\sigma}(f) \leq C_{0} K_{i} M_{i, j} p_{j}(f)=C_{0} L_{i, j} p_{j}(f), \quad \forall f \in E .
$$

So we deduce that $q^{\sigma}$ is continuous on $E$ and hence Theorem 17 implies that $\left(P_{k}\right)_{k \geq 0}$ is effective for $E$ in $F$. Theorem 18 is therefore established.

\section{Applications}

We need to mention some definitions and notations in Clifford analysis [21, 22, 33, 34].

Definition 19 (monogenic function). Let $\Omega \subset \mathbb{R}^{m+1}$ be an open set; then an $\mathscr{A}_{m}$-valued function $f$ is called left (resp. right) monogenic in $\Omega$ if it satisfies $D f=0$ (resp. $f D=0$ ) in $\Omega$. Here, $D=\sum_{i=0}^{m} e_{i}\left(\partial / \partial x_{i}\right)$, defined in Section 2, is the generalized Cauchy-Riemann operator.

Definition 20 (special monogenic polynomial). A polynomial $P(x)$ is special monogenic if and only if $D P(x)=0$ (so $P(x)$ is monogenic) and there exists $a_{i, j} \in \mathscr{A}_{m}$ for which $P(x)=$ $\sum_{i, j}^{\text {finite }} \bar{x}^{i} x^{j} a_{i, j}$.

Definition 21 (special monogenic function). Let $\Omega$ be a connected open subset of $\mathbb{R}^{m+1}$ containing 0 and let $f$ be monogenic in $\Omega$. Then $f$ is called special monogenic in $\Omega$ if and only if its Taylor series near zero (which exists) has the form $f(x)=\sum_{n=0}^{\infty} P_{n}(x)$ for certain special monogenic polynomials $P_{n}(x)$.

The fundamental references for special monogenic function are $[36,37]$.

Remark 22. Note that if $P_{n}(x)$ is a homogeneous special monogenic polynomial of degree $n$, then (see $[21,29]) P_{n}(x)=$ $\mathscr{P}_{n}(x) \alpha: \alpha$ is some constant in $\mathscr{A}_{m}$ and

$$
\begin{array}{r}
\mathscr{P}_{n}(x)=\frac{n !}{(m)_{n}} \sum_{k+l=n} \frac{((m-1) / 2)_{k}}{k !} \frac{((m+1) / 2)_{l}}{l !} \bar{x}^{k} x^{l}, \\
x \in \mathbb{R}^{m+1},
\end{array}
$$

where, for $b \in \mathbb{R},(b)_{l}=b(b+1) \cdots(b+l-1)$.

It is well known that $\left(\mathscr{P}_{n}(x)\right)_{n \geq 0}$ is an Appell sequence with respect to $\partial / \partial x_{0}$ or $(1 / 2) \bar{D}$ (which represent the same operator for monogenic functions): $(1 / 2) \bar{D} \mathscr{P}_{n}(x)=$ $n \mathscr{P}_{n-1}(x)$ and $\bar{D}=\sum_{i=0}^{m} \bar{e}_{i}\left(\partial / \partial x_{i}\right)$ in $\mathbb{R}^{m+1}$ (see [38-40]). [21])

The maximum value of $\left|\mathscr{P}_{n}(x)\right|$ in $|x|=R$ is given by (see

$$
\left\|\mathscr{P}_{n}(x)\right\|_{R}:=\sup _{|x|=R}\left|\mathscr{P}_{n}(x)\right|=R^{n}
$$

An open ball is usually denoted by $B(r)$, and closed ball is denoted by $\bar{B}(r)$, where

$$
\begin{aligned}
& B(r)=\left\{x \in \mathbb{R}^{m+1},|x|<r\right\}, \\
& \bar{B}(r)=\left\{x \in \mathbb{R}^{m+1},|x| \leq r\right\} .
\end{aligned}
$$

Also, the class of special monogenic functions in an open ball $B(r)$ is written as $H(r)$ and $\bar{H}(r)$ denotes the class of special monogenic functions in closed ball $\bar{B}(r)$.

The first application of the above theory is to the effectiveness in an open ball.

7.1. Effectiveness in Open Balls. We propose to derive in the present section, from the results of Section 5, conditions for effectiveness of basic sets in open balls. For this case, we take the Fréchet module $E$ to be the class $H(r), r>0$, of special monogenic functions in the open ball $B(r)$.

Let $\rho_{0}$ be a certain positive number less than $r$ and construct the sequence $\left(\rho_{n}\right)_{n \geq 0}$ as follows:

$$
\begin{gathered}
\rho_{1}=\frac{r+\rho_{0}}{2}, \\
\rho_{2}=\frac{r+\rho_{1}}{2}, \\
\vdots \\
\rho_{n+1}=\frac{r+\rho_{n}}{2},
\end{gathered}
$$

So

$$
\rho_{0}<\rho_{1}<\rho_{2}<\cdots<\rho_{n}<\cdots<r .
$$

The countable family $\mathscr{P}$ of seminorms $\left(p_{i}\right)_{i \geq 0}$ on the Fréchet module $H(r)$ is defined as follows.

For $f \in H(r)$, we set

$$
p_{i}(f)=\sup _{\bar{B}\left(\rho_{i}\right)}|f(x)|=M\left(f, \rho_{i}\right) .
$$

Thus, when $i<j, \rho_{i}<\rho_{j}$. So

$$
p_{i}(f) \leq p_{j}(f) ; \quad \forall f \in H(r),
$$

and, therefore, condition (i) of Definition 5 is satisfied.

The topology on $H(r)$ defined by the family $\left(p_{i}\right)_{i \geq 0}$ is the topology of normal convergence over the compact sets $\bar{B}\left(\rho_{i}\right), i \geq 0$. It is easy to show that the $\mathscr{A}_{m}$-module $H(r)$ is complete for this topology; that is to say, $H(r)$ is a Fréchet module.

We shall take a basis for $H(r)$, the Appell sequence $\left(\mathscr{P}_{n}(x)\right)_{n \geq 0}$. In fact every function $f \in H(r)$ has the unique expansion

$$
f(x)=\sum_{n=0}^{\infty} \mathscr{P}_{n}(x) a_{n}(f) .
$$

Thus (7) is true. In this case, Cauchy's inequality (8) takes the form (see [22])

$$
\left|a_{n}(f)\right| \rho_{i}^{n} \leq \sup _{\bar{B}\left(\rho_{i}\right)}|f(x)|=\|f\|_{\rho_{i}} .
$$


It can be verified also that $\left(\mathscr{P}_{n}(x)\right)_{n \geq 0}$ is an absolute basis for $H(r)$ in the sense that series (11), which is rewritten here as

$$
\sum_{n=0}^{\infty}\left|a_{n}(f)\right| \rho_{i}^{n}
$$

is convergent $\forall i \geq 0$.

Finally, when $i<j, \rho_{i}<\rho_{j}$, so

$$
\sum_{n=0}^{\infty} \frac{p_{i}\left(\mathscr{P}_{n}(x)\right)}{p_{j}\left(\mathscr{P}_{n}(x)\right)}=\sum_{n=0}^{\infty} \frac{\rho_{i}^{n}}{\rho_{j}^{n}}<\infty
$$

and then relation (9) holds.

Now, let $\left(P_{k}\right)_{k \geq 0}=\left(P_{k}(x)\right)_{k \geq 0}$ be a basic set. Expression (18) is the unique representation

$$
\mathscr{P}_{n}(x)=\sum_{k=0}^{\infty} P_{k}(x) \pi_{n, k}
$$

and if $f \in H(r)$, then by substituting (63) in (59) we obtain the basic series of $f$ :

$$
f(x) \sim \sum_{k=0}^{\infty} P_{k}(x) \Pi_{k}(f)
$$

where

$$
\Pi_{k}(f)=\sum_{n=0}^{\infty} \pi_{n, k} a_{n}(f) ; \quad(k \geq 0)
$$

is the basic coefficient of $f$.

In this case, the expression $q_{i}\left(\mathscr{P}_{n}(x)\right)$ is called the Cannon sum for the set $\left(P_{k}\right)$ and is denoted by $F_{n}\left(\rho_{i}\right)$ :

$$
q_{i}\left(\mathscr{P}_{n}(x)\right)=\sup _{\mu, \nu} \sup _{\bar{B}\left(\rho_{i}\right)}\left|\sum_{k=\mu}^{v} P_{k}(x) \pi_{n, k}\right|=F_{n}\left(\rho_{i}\right) .
$$

The Cannon function for the same set in $\bar{B}(\rho)$ is

$$
K(\rho)=\limsup _{n \rightarrow \infty}\left(F_{n}(\rho)\right)^{1 / n} .
$$

It should be observed that (63), (66), and (67) together yield

$$
K(\rho) \geq \rho
$$

and it is easily seen that $K(\rho)$ is a monotonic increasing function of $\rho$.

The fundamental theorem for effectiveness for $H(r)$ is deducible from Theorem 15. It is stated in the following form.

Theorem 23. The necessary and sufficient condition for the basic set $\left(P_{k}(x)\right)_{k \geq 0}$ to be effective for $H(r)$ is that

$$
K(\rho)<r, \quad \forall \rho<r .
$$

Proof. Suppose that $\rho$ is any positive number less than $r$; then there exists a number $\rho_{i}$ such that

$$
\rho \leq \rho_{i}<r .
$$

If the set $\left(P_{k}(x)\right)_{k \geq 0}$ is effective for $H(r)$, then, by Theorem 15, there exist $\rho_{j}<r$ and a constant $K_{i, j}$ such that

$$
q_{i}\left(\mathscr{P}_{n}(x)\right) \leq K_{i, j} p_{j}\left(\mathscr{P}_{n}(x)\right) ; \quad \forall n \in \mathbb{N} .
$$

Hence, (66) gives

$$
F_{n}\left(\rho_{i}\right) \leq K_{i, j} \rho_{j}^{n}
$$

Using (67) and (70), we can deduce that

$$
K(\rho) \leq K\left(\rho_{i}\right) \leq \rho_{j}<r
$$

and condition (69) follows. Thus (69) is necessary.

On the other hand, suppose that condition (69) is satisfied and let $\rho_{i}$ be any element of sequence (56). So we have

$$
K\left(\rho_{i}\right)<r .
$$

Since the sequence $\left(\rho_{i}\right)_{i \geq 0}$ converges to $r$ as $i$ tends to infinity, then there exists an integer $j>i$ such that

$$
K\left(\rho_{i}\right)<\rho_{j}<r .
$$

Then, by definition (67) of $K(\rho)$, there exists $C_{i, j}$ such that

$$
F_{n}\left(\rho_{i}\right) \leq C_{i, j} \rho_{j}^{n}
$$

Applying (53), (57), and (66), it follows that

$$
q_{i}\left(\mathscr{P}_{n}(x)\right) \leq C_{i, j} p_{j}\left(\mathscr{P}_{n}(x)\right) ; \quad \forall n \in \mathbb{N} .
$$

Hence, by Theorem 15 , the set $\left(P_{k}\right)_{k \geq 0}$ is effective for $H(r)$, and the theorem is satisfied.

7.2. Effectiveness in Closed Balls. Let $R$ be any fixed positive number and take the number $r$ to be any finite number greater than $R$. The $\mathscr{A}_{m}$-module $F$ of Section 6 will be taken as the class $\bar{H}(R)$ of special monogenic functions in the closed ball $\bar{B}(R)$, with the norm $\sigma$ defined by

$$
\sigma(f)=\sup _{\bar{B}(R)}|f(x)|=M(f ; R), \quad \forall f \in \bar{H}(R) .
$$

Thus, the topology $\mathscr{T}_{\bar{H}(R)}$ determined by the norm $\sigma$ is the topology of normal convergence on $\bar{B}(R)$. It is well known that $\bar{H}(R)$ is complete for this topology; that is to say, $\bar{H}(R)$ is a Banach module. The subspace $E$ of $\bar{H}(R)$ will be taken as the Fréchet module $H(r)$ (see Section 7.1) which will be equipped with the family of seminorms $\left(p_{i}\right)_{k \geq 0}$ defined by

$$
p_{i}(f)=M\left(f, \rho_{i}\right) ; \quad i \in \mathbb{N}, f \in H(r),
$$

where

$$
\begin{gathered}
\rho_{0}=R, \\
\rho_{1}=\frac{r+\rho_{0}}{2}, \\
\rho_{2}=\frac{r+\rho_{1}}{2},
\end{gathered}
$$

$$
\rho_{n+1}=\frac{r+\rho_{n}}{2}
$$


It is clear that

$$
\sigma(f) \leq p_{i}(f) ; \quad i \in \mathbb{N}, f \in H(r) .
$$

So condition (40) is satisfied.

The basis for $H(r)$ is taken, as before, to be the Appell sequence $\left(\mathscr{P}_{n}(x)\right)_{n \geq 0}$ which accords to Cauchy's inequality and it is also an absolute basis. Moreover, condition (9) is satisfied.

Now, let $\left(P_{k}(x)\right)_{k \geq 0}$ be a basic set of $\bar{H}(R)$ and suppose that $\mathscr{P}_{n}(x)$ admits the representation

$$
\mathscr{P}_{n}(x)=\sum_{k=0}^{\infty} P_{k}(x) \pi_{n, k}
$$

where the convergence is in $\bar{H}(R)$, so that

$$
\max _{\bar{B}(R)}\left|\mathscr{P}_{n}(x)-\sum_{k=0}^{m} P_{k}(x) \pi_{n, k}\right| \longrightarrow 0, \quad \text { as } m \longrightarrow \infty .
$$

Write the basic coefficient and the basic series as in (64) and (65). We are concerned with the convergence in $\bar{H}(R)$; that is to say, basic series (64) converges to $f$ in $\bar{H}(R)$ if

$$
\sup _{\bar{B}(R)}\left|f(x)-\sum_{k=0}^{m} P_{k}(x) \Pi_{k}(f)\right| \longrightarrow 0, \quad \text { as } m \longrightarrow \infty .
$$

The basic set $\left(P_{k}\right)_{k \geq 0}$ will be effective for $H(r)$ in $\bar{H}(R)$ if the basic series of each special monogenic function in $B(r)$ converges to $f$ normally in $\bar{B}(R)$. In this case, we say that the basic set $\left(P_{k}\right)_{k \geq 0}$ is effective for $H(r)$ in $\bar{B}(R), R<r$.

The theorem about such effectiveness is deducible from Theorem 18.

As in (66), we can see, by using (78), that

$$
q^{\sigma}\left(\mathscr{P}_{n}(x)\right)=F_{n}(R) .
$$

We shall establish the following Theorem.

Theorem 24. The necessary and sufficient condition for the basic set $\left(P_{k}(x)\right)_{k \geq 0}$ to be effective for $H(r)$ in $\bar{B}(R), r>R>0$, is that

$$
K(R)<r .
$$

Proof. Suppose that $\left(P_{k}\right)_{k \geq 0}$ is effective for $H(r)$ in $\bar{B}(R)$. Hence, according to Theorem 18, there exist a norm $p_{i} \in \mathscr{P}$ and a constant $K_{i}$ such that

$$
q^{\sigma}\left(\mathscr{P}_{n}(x)\right) \leq K_{i} p_{i}\left(\mathscr{P}_{n}(x)\right), \quad n \geq 0 .
$$

Hence, (67), (79), and (85) together yield

$$
K(R) \leq \rho_{i}<r
$$

and condition (86) is necessary.

On the other hand, suppose that condition (86) is satisfied. Since $\rho_{n} \rightarrow r$ as $n \rightarrow \infty$, there is a number $\rho_{i}$ such that
$K(R)<\rho_{i}<r$. Then, by definition (67) of $K(\rho)$, there exists $K_{i}$ such that

$$
F_{n}(R) \leq K_{i} \rho_{i}^{n}, \quad n \geq 0 .
$$

Hence, from (53), (57), and, (85) it follows that

$$
q^{\sigma}\left(\mathscr{P}_{n}(x)\right) \leq K_{i} p_{\mathrm{i}}\left(\mathscr{P}_{n}(x)\right), \quad n \geq 0
$$

By Theorem 18, it follows that the basic set $\left(P_{k}(x)\right)_{k \geq 0}$ is effective for $H(r)$ in $\bar{B}(R)$ as required.

Taking $r$ sufficiently near to $R$, Theorem 24 leads to the following corollary.

Corollary 25. The necessary and sufficient condition for the basic set $\left(P_{k}(x)\right)_{k \geq 0}$ to be effective in $\bar{B}(R)$ is that

$$
K(R)=R \text {. }
$$

7.3. Cannon Sets. When $\left(P_{k}\right)_{k \geq 0}$ is a basic set of special monogenic polynomials, representation (63) is finite. Thus, if $N_{n}$ is the number of nonzero coefficients in (63), then $N_{n}$ is finite. If this number accords further to the restriction that

$$
N_{n}^{1 / n} \longrightarrow 1, \quad \text { as } n \longrightarrow \infty,
$$

the corresponding basic set $\left(P_{k}(x)\right)_{k \geq 0}$ of polynomials is called Cannon set of special monogenic polynomials (see [21]). Write

$$
\begin{aligned}
\omega_{n}(\rho) & =\sum_{k}\left\|P_{k}(x) \pi_{n, k}\right\|_{\rho} \\
& :=\sum_{k} \sup _{\bar{B}(\rho)}\left|P_{k}(x) \pi_{n, k}\right| ; \quad(\rho>0), \\
\left\|P_{s_{n}}(x) \pi_{n, s_{n}}\right\|_{\rho} & =\max _{k}\left\|P_{k}(x) \pi_{n, k}\right\|_{\rho} .
\end{aligned}
$$

Then, in view of (66), we shall have

$$
\begin{aligned}
\left\|P_{s_{n}}(x) \pi_{n, s_{n}}\right\|_{\rho} & \leq F_{n}(\rho) \leq \omega_{n}(\rho) \\
& \leq N_{n}\left\|P_{s_{n}}(x) \pi_{n, s_{n}}\right\|_{\rho} .
\end{aligned}
$$

Hence, if we put

$$
\lambda(\rho)=\limsup _{n \rightarrow \infty}\left(\omega_{n}(\rho)\right)^{1 / n},
$$

then, for Cannon sets, relation (67) implies that

$$
\lambda(\rho)=K(\rho) .
$$

Therefore, for Cannon sets, the Cannon sum and the Cannon function $\omega_{n}(\rho)$ and $\lambda(\rho)$ are given by (93) and (96), respectively.

For Cannon sets, the Cannon function $\lambda(\rho)$ will replace $K(\rho)$ in all the concerned relations: (69), (86), and (91). Hence, concerning the effectiveness of Cannon sets of special monogenic polynomials (see $[21,23]$ ), we have the following results which are special cases of our results. 
Corollary 26. The necessary and sufficient condition for the Cannon sets of special monogenic polynomials $\left(P_{k}(x)\right)_{k \geq 0}$ to be effective for $H(r)$ is that

$$
\lambda(\rho)<r, \quad \forall \rho<r .
$$

Corollary 27. The necessary and sufficient condition for the Cannon sets of special monogenic polynomials $\left(P_{k}(x)\right)_{k \geq 0}$ to be effective for $H(r)$ in $\bar{B}(R), r>R>0$, is that

$$
\lambda(R)<r .
$$

Corollary 28. The necessary and sufficient condition for the Cannon sets of special monogenic polynomials $\left(P_{k}(x)\right)_{k \geq 0}$ to be effective for in $\bar{B}(R)$ is that

$$
\lambda(R)=R .
$$

\section{Disclosure}

The current address of Gamal Farghaly Hassan is "Department of Mathematics, Faculty of Sciences, Northern Border University, P.O. Box 1321, Arar, Saudi Arabia”.

\section{Competing Interests}

The authors declare that they have no competing interests.

\section{Acknowledgments}

The authors wish to acknowledge the approval and the support of this research study from the Deanship of Scientific Research in Northern Border University, Arar, Saudi Arabia (Grant no. 5-7-1436-5).

\section{References}

[1] J. M. Whittaker, "On series of polynomials," The Quarterly Journal of Mathematics, vol. os-5, no. 1, pp. 224-239, 1934.

[2] J. M. Whittaker, Sur les Séries de Base de Polynômes Quelconques. Avec la collaboration de c. Gattegno (Collection de monographies sur la theorie des fonctions), Gauthier-Villars, Paris, France, 1949.

[3] M. A. Abul-Ez, "Bessel polynomial expansions in spaces of holomorphic functions," Journal of Mathematical Analysis and Applications, vol. 221, no. 1, pp. 177-190, 1998.

[4] L. Aloui, M. A. Abul-Ez, and G. F. Hassan, "Bernoulli special monogenic polynomials with the difference and sum polynomial bases," Complex Variables and Elliptic Equations, vol. 59, no. 5, pp. 631-650, 2014.

[5] L. Aloui, M. A. Abul-Ez, and G. F. Hassan, "On the order of the difference and sum bases of polynomials in Clifford setting," Complex Variables and Elliptic Equations. An International Journal, vol. 55, no. 12, pp. 1117-1130, 2010.

[6] R. P. Boas Jr. and R. B. Buck, Polynomial Expansions of Analytic Functions, Springer, Berlin, Germany, 1964.

[7] G. F. Hassan and L. Aloui, "Bernoulli and Euler polynomials in Clifford analysis," Advances in Applied Clifford Algebras, vol. 25, no. 2, pp. 351-376, 2015.

[8] B. Cannon, "On the convergence of series of polynomials," Proceedings of the London Mathemaical Society, vol. 43, pp. 348364, 1937.
[9] B. Cannon, "On the convergence of integral functions by general basic series," Mathematische Zeitschrift, vol. 45, pp. 158-205, 1939.

[10] M. Mursi and R. H. Maker, "Bases of polynomials of several complex variables I.II," in Proceedings of the 2nd Arab Science Congress, vol. 2, pp. 51-68, Cairo, Egypt, September 1955.

[11] M. Nassif, "Composite sets of polynomials of several complex variables," Publicationes Mathematicae Debrecen, vol. 18, pp. 43$52,1971$.

[12] A. El-Sayed and Z. G. Kishka, "On the effectiveness of basic sets of polynomials of several complex variables in elliptical regions," in Proceedings of the 3rd International ISAAC Congress, pp. 265-278, Freie Universitaet, Berlin, Germany, 2003.

[13] G. F. Hassan, "Ruscheweyh differential operator sets of basic sets of polynomials of several complex variables in hyperelliptical regions," Acta Mathematica Academiae Paedagogicae Nyiregyhaziensis, vol. 22, no. 3, pp. 247-264, 2006.

[14] Z. G. Kishka, M. A. Saleem, and M. A. Abul-Dahab, "On simple exponential sets of polynomials," Mediterranean Journal of Mathematics, vol. 11, no. 2, pp. 337-347, 2014.

[15] W. F. Kumuyi and M. Nassif, "Derived and integrated sets of simple sets of polynomials in two complex variables," Journal of Approximation Theory, vol. 47, no. 4, pp. 270-283, 1986.

[16] R. H. Makar and L. Fawzy, "On the effectiveness of base sets of polynomials associayed with functions of algebraic infinite matrices," Publicationes Mathematicae Debrecen, Hungaria, vol. 25, pp. 73-76, 1978.

[17] M. N. Mikhail, "Simple basic sets of polynomials," American Journal of Mathematics, vol. 76, pp. 647-653, 1954.

[18] M. N. Mikhail, "The square root set of a simple basic set of polynomials," Duke Mathematical Journal, vol. 25, pp. 177-180, 1958.

[19] W. F. Newns, "A note on basic sets of polynomials," Duke Mathematical Journal, vol. 18, pp. 735-739, 1951.

[20] W. F. Newns, "On the representation of analytic functions by infinite series," Philosophical Transactions of the Royal Society of London. Series A. Mathematical and Physical Sciences, vol. 245, pp. $429-468,1953$.

[21] M. A. Abul-Ez and D. Constales, "Basic sets of polynomials in Clifford analysis," Complex Variables and Elliptic Equations, vol. 14, pp. 177-185, 1990.

[22] M. A. Abul-Ez and D. Constales, "Linear substitution for basic sets of polynomials in Clifford analysis," Portugaliae Mathematica, vol. 48, no. 2, pp. 143-154, 1991.

[23] M. A. Abul-Ez and M. Zayad, "Similar transposed bases of polynomials in clifford analysis," Applied Mathematics and Information Sciences, vol. 4, pp. 63-78, 2010.

[24] M. A. Abul-Ez and D. Constales, "On the order of basic series representing Clifford valued functions," Applied Mathematics and Computation, vol. 142, no. 2-3, pp. 575-584, 2003.

[25] M. A. Abul-Ez and D. Constales, "The square root base of polynomials in clifford analysis," Archiv der Mathematik, vol. 80, no. 5, pp. 486-495, 2003.

[26] M. A. Abul-Ez, D. Constales, J. Morais, and M. Zayed, "Hadamard three-hyperballs type theorem and overconvergence of special monogenic simple series," Journal of Mathematical Analysis and Applications, vol. 412, no. 1, pp. 426-434, 2014.

[27] L. Aloui and G. F. Hassan, "Hypercomplex derivative bases of polynomials in Clifford analysis," Mathematical Methods in the Applied Sciences, vol. 33, no. 3, pp. 350-357, 2010. 
[28] G. F. Hassan, "A note on the growth order of the inverse and product bases of special monogenic polynomials," Mathematical Methods in the Applied Sciences, vol. 35, no. 3, pp. 286-292, 2012.

[29] M. Zayed, M. A. Abul-Ez, and J. P. Morais, "Generalized derivative and primitive of Cliffordian bases of polynomials constructed through Appell monomials," Computational Methods and Function Theory, vol. 12, no. 2, pp. 501-515, 2012.

[30] M. A. Saleem, M. Abul-Ez, and M. Zayed, "On polynomial series expansions of Cliffordian functions," Mathematical Methods in the Applied Sciences, vol. 35, no. 2, pp. 134-143, 2012.

[31] J. A. Adepoju, Basic sets of Gonarov polynomials and Faber regions [Ph.D. thesis], University of Lagos, Lagos, Nigeria, 1979.

[32] A. El-Sayed Ahmed, On some classes and spaces of holomorphic and hyperholomorphic functions [Ph.D. thesis], Bauhaus University, Weimar, Germany, 2003.

[33] F. Brackx, R. Delanghe, and F. Sommen, Clifford Analysis (Research Notes in Mathematics, 76), Pitman Advanced Publishing Program, London, UK, 1st edition, 1982.

[34] K. Gürlebeck, K. Habetha, and W. Sprössig, Holomorphic Functions in the Plane and n-Dimensional Space, Birkhäuser, Basel, Switzerland, 2008.

[35] G. Grubb, Distributions and Operators, Springer, Berlin, Germany, 2009.

[36] P. Lounesto and P. Bergh, "Axially symmetric vector fields and their complex potentials," Complex Variables and Elliptic Equations, vol. 2, pp. 139-150, 1983.

[37] F. Sommen, "Plane elliplic systems and monogenic functions in symmetric domains," Rendiconti del Circolo Matematico di Palermo, vol. 6, pp. 259-269, 1984.

[38] M. I. Falcäo, J. F. Cruz, and H. R. Malonek, "Remarks on the generation of monogenic functions," in Proceedings of the 17th International Conference on the Application of Computer Science and Mathematics in Architecture and Civil Engineering, $\mathrm{K}$. Gürlebeck and C. Könke, Eds., Weimar, Germany, July 2006.

[39] M. I. Falcäo and H. R. Malonek, "Generalized exponentials through Appell sets in $\mathrm{Rn}+1$ and Bessel functions," in Proceedings of the Internatonal Conference on Numerical Analysis and Applied Mathematics, T. Simos, G. Psihoyios, and Ch. Tsitouras, Eds., pp. 738-741, Corfu, Greece, September 2007.

[40] N. Gürlebeck, "On Appell sets and the Fueter-Sce mapping," Advances in Applied Clifford Algebras, vol. 19, no. 1, pp. 51-61, 2009. 


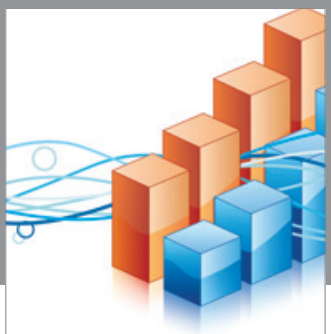

Advances in

Operations Research

vatem alat4

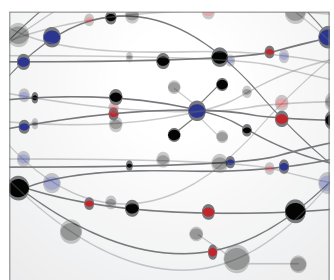

\section{The Scientific} World Journal
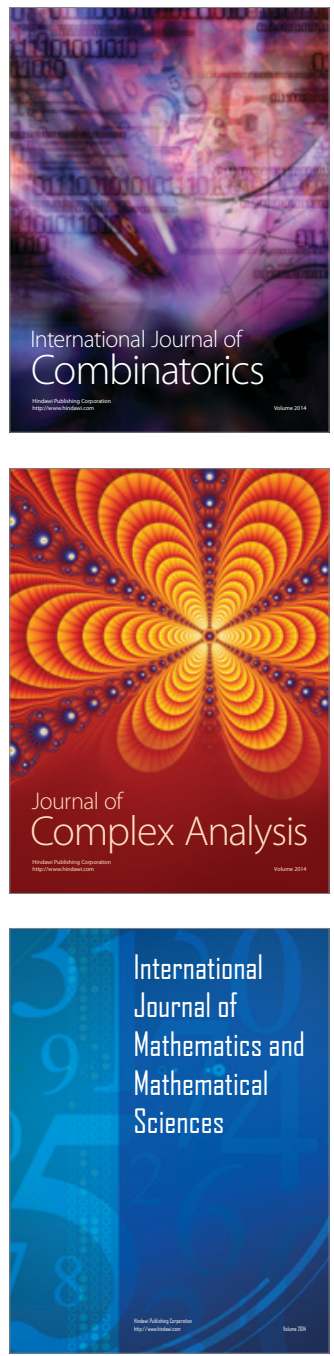
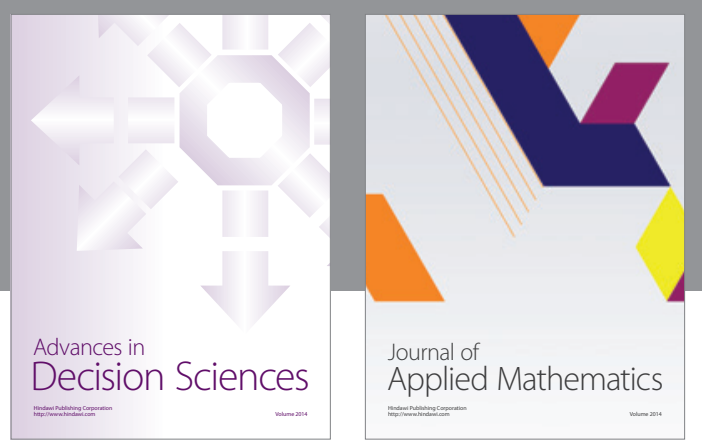

Algebra

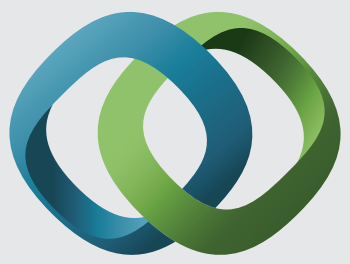

\section{Hindawi}

Submit your manuscripts at

https://www.hindawi.com
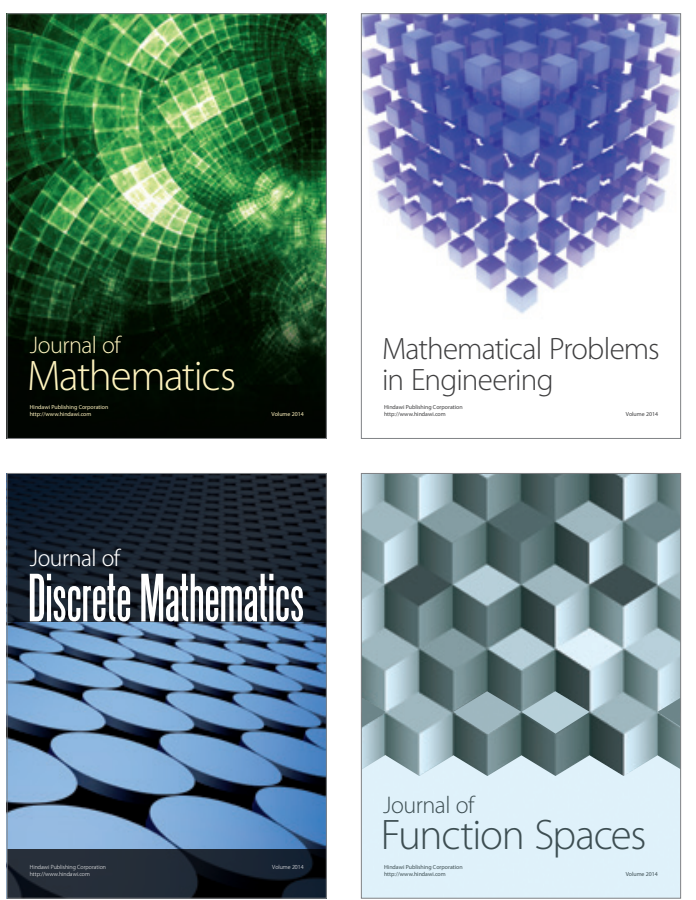

Mathematical Problems in Engineering
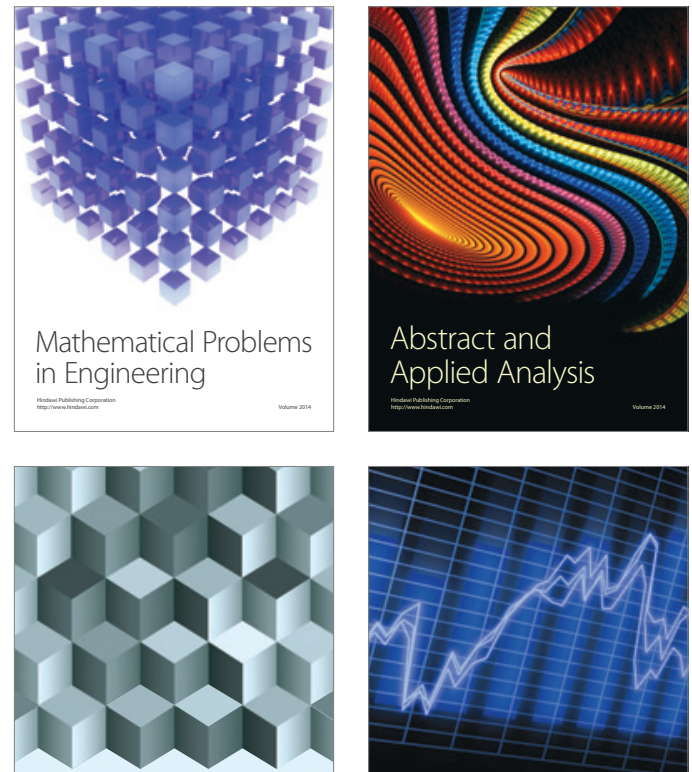

Journal of

Function Spaces

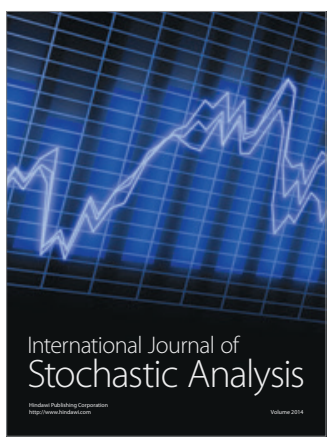

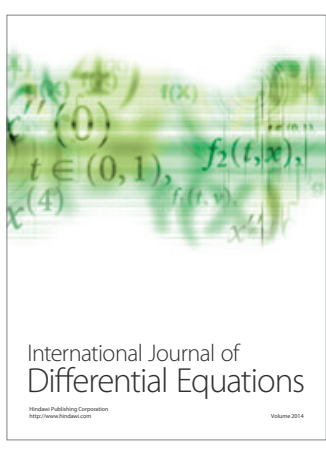
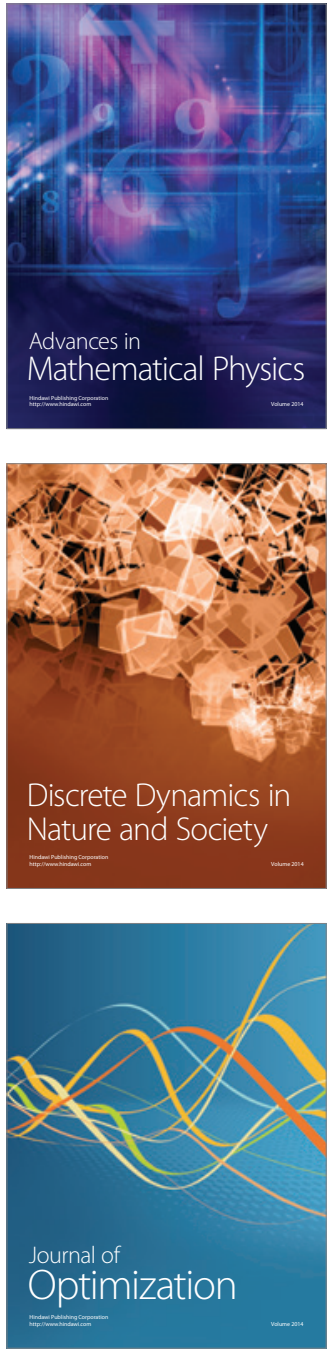